\title{
TV/Series
}

\section{The West Wing et la Constitution des États-Unis}

\section{Damien Connil}

\section{(2) OpenEdition}

\section{Journals}

Édition électronique

URL : http://journals.openedition.org/tvseries/648

DOI : 10.4000/tvseries.648

ISSN : 2266-0909

Éditeur

GRIC - Groupe de recherche Identités et Cultures

\section{Référence électronique}

Damien Connil, «The West Wing et la Constitution des États-Unis », TV/Series [En ligne], 8| 2015, mis en ligne le 01 décembre 2015, consulté le 05 mai 2019. URL : http://journals.openedition.org/ tvseries/648; DOI : 10.4000/tvseries.648

Ce document a été généré automatiquement le 5 mai 2019.



TV/Series est mis à disposition selon les termes de la licence Creative Commons Attribution - Pas d'Utilisation Commerciale - Pas de Modification 4.0 International. 


\title{
The West Wing et la Constitution des États-Unis
}

\author{
Damien Connil
}

1 The West Wing raconte le quotidien du pouvoir présidentiel aux États-Unis, ses moyens et ses limites, ses partenaires et ses opposants, ce qu'il doit faire et ce qu'il peut faire. Du point de vue du droit, cela renvoie directement à la règle fondatrice de la vie institutionnelle, autrement dit, la Constitution et, plus largement, le droit constitutionnel. La série n'évite pas la question. Bien au contraire, la Constitution est citée à plusieurs reprises, des mécanismes et des procédures constitutionnels sont invoqués, mis en scène et expliqués.

2 Au fil des épisodes sont, par exemple, évoqués : la protection des libertés individuelles ; le veto législatif du Président; l'obstruction parlementaire; la prestation de serment du Président élu; l'adoption du budget fédéral; la mise en jeu de la responsabilité du Président; les pouvoirs du Vice-Président; le rôle des commissions parlementaires; la désignation des juges de la Cour suprême ou encore le pouvoir de grâce du Président ${ }^{1}$.

C'est précisément sur cette diversité des situations constitutionnelles et leur mise à l'écran que nous voudrions nous appuyer pour souligner à quel point la série est un extraordinaire instrument d'initiation au droit constitutionnel des États-Unis. Il s'agit d'observer les images proposées par The West Wing de la Constitution, d'apprécier ce que cette représentation nous permet de comprendre de la réalité du droit constitutionnel et d'analyser comment cette mise en scène participe aussi à la construction d'un imaginaire constitutionnel.

4 Deux précautions de méthode sont alors indispensables. Primo, l'objet de l'étude est d'observer de manière globale comment, au cours de ses sept saisons, The West Wing, aborde, suggère ou évoque le droit constitutionnel. Une analyse d'ensemble sera menée un tableau constitutionnel brossé à grands traits - et non une étude exhaustive du droit constitutionnel tel que mis en scène dans la série qui dépasserait largement le cadre nécessairement restreint de cet article. Par conséquent, seuls quelques exemples, particulièrement significatifs de la représentation à l'écran, dans la série, de la Constitution et du droit constitutionnel seront choisis et utilisés pour illustrer le propos, 
un certain nombre d'entre eux pouvant d'ailleurs être évoqués à plusieurs reprises. Cependant, c'est bien une vision d'ensemble de la question que nous souhaitons mettre en évidence. Par ailleurs, l'étude se place résolument du point de vue du constitutionnaliste même si le détail juridique ou technique de certains mécanismes ou procédures n'est pas analysé dès lors que là n'est pas l'essentiel. Le but n'est pas d'examiner en détail le droit constitutionnel dans The West Wing mais de souligner la possibilité pour le spectateur d'appréhender des questions d'ordre constitutionnel à travers les épisodes de la série.

Pour cela, examinons successivement les images (I), les explications (II) et les représentations (III) que The West Wing propose de la Constitution et du droit constitutionnel.

\section{Des images}

\section{La mise en image du droit constitutionnel et de sa pratique}

Tout d'abord, les personnages de la série vivent et mettent en œuvre le droit constitutionnel. Ils montrent aux spectateurs le droit constitutionnel en train de se vivre et de se faire et offrent ainsi à voir ce qu'il est ou ce qu'il peut être. C'est de cette manière que la série met alors en « images » ces règles de droit et leur pratique.

7 À plusieurs reprises, par exemple, la question de l'interprétation d'une disposition constitutionnelle se trouve au cœur de la narration. Qu'il s'agisse de savoir si le droit à la vie privée est garanti par la Constitution ${ }^{2}$ ou qu'il s'agisse de savoir ce qu'il faut entendre exactement par l'expression "from time to time » ("de temps en temps») à propos du discours sur l'état de l'Union qui doit être délivré selon l'Article II, Section 3 de la Constitution " périodiquement ${ }^{3}$.» D'autres exemples pourraient encore être évoqués, et c'est bien dans tous les cas la question de l'interprétation du texte constitutionnel qui est portée à l'écran. La série montre alors comment une question d'ordre constitutionnel apparaît et se pose au Président et à ses conseillers.

8 The West Wing est remarquable non seulement parce que, de cette manière, une série télévisée place au cœur de l'intrigue une question qui est elle-même au cœur des interrogations les plus fondamentales des spécialistes de droit constitutionnel, mais aussi parce qu'ainsi, le texte même de la Constitution est cité, discuté, débattu dans une œuvre de divertissement et à une heure de grande écoute.

\section{La mise en scène du phénomène constitutionnel}

9 La série offre une véritable mise en scène du phénomène constitutionnel. À cet égard, les deux épisodes de la saison 5 ( 5.7 et 5.8 ) consacrés à l'élaboration du budget fédéral sont significatifs ${ }^{4}$. On ne peut pas dire que le vote du budget soit un sujet particulièrement télégénique. Pourtant, en s'appuyant sur la réalité et en présentant ce processus de manière très accessible, The West Wing parvient à mettre en scène et à dramatiser cet événement constitutionnel et parlementaire particulier qu'est l'adoption du budget. Elle transforme même l'élaboration du budget fédéral en véritable thriller ${ }^{5}$.

10 Il faut dire que l'adoption du budget présente, en elle-même, un intérêt dramaturgique évident aux États-Unis puisque dans un contexte de gouvernement divisé (divided governement) où exécutif et législatif ne sont pas de la même couleur politique, rien 
n'illustre mieux les tensions qui opposent le Congrès au Président que l'élaboration du budget fédéral. Il n'en demeure pas moins qu'il y a, dans la série, une véritable mise en scène d'un événement qui relève du droit constitutionnel et une véritable dramatisation de cet événement. Surtout qu'il faut ajouter aux dialogues et à l'intrigue (qui se déroule sur deux épisodes), les éléments classiques du film à suspense : la musique, les ralentis et les gros plans qui soulignent les tensions entre exécutif et législatif, comme l'a montré Marjolaine Boutet $^{6}$.

11 Des événements constitutionnels sont ainsi mis en scène et on pourrait également évoquer les discours sur l'état de l'Union, la rentrée solennelle de la Cour suprême, la prestation de serment du Président élu et bien d'autres encore ${ }^{7}$. The West Wing donne aussi à voir de manière différente le droit constitutionnel en ce qu'elle en offre une vision inédite. La série nous permet de voir ce que l'on ne peut, normalement, pas voir.

\section{L'exploration des coulisses}

12 La série nous donne, par exemple, l'occasion d'assister à des réunions dans la salle des crises où sont abordées des questions qui mettent en jeu des éléments d'ordre constitutionnel: les pouvoirs du Président (et ses rapports avec le Congrès), la lutte contre le terrorisme (et la protection des droits fondamentaux des individus), les opérations militaires en cours (et des questions de respect de souveraineté étatique et de déploiement des forces armées à l'étranger ${ }^{8}$ ). Là encore, la multiplication des situations qui touchent à des questions constitutionnelles participe d'une mise en images et en pratique (une pratique cependant fictionnelle) du droit.

Charles Girard, dans un article consacré à la place de la parole dans la série, observe très justement que «The West Wing ne s'attarde guère sur les faces connues de la présidence : les épisodes s'achèvent alors qu'un discours présidentiel va commencer, ou débutent quand il s'éloigne déjà de la tribune. Ce sont les coulisses ou plutôt les couloirs du pouvoir exécutif, rarement visibles à l'écran, que la fiction prétend mettre au jour'. "

Cela se vérifie presque à chaque fois qu'un événement constitutionnel est convoqué dans

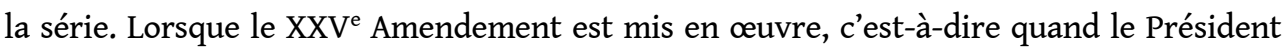
Bartlet se retire temporairement de la présidence, au moment de l'enlèvement de sa fille Zoé, c'est ce que l'on ne peut normalement pas voir qui est montré dans la série: les discussions entre les conseillers qui font part aussi bien de leurs arguments que de leurs craintes; la signature dans le Bureau ovale du document officiel; les conflits entre les différentes équipes, etc ${ }^{10}$.

Lorsque le Président Bartlet décide d'opposer son veto à un texte adopté par le Congrès, ce sont, là encore, les coulisses de cette procédure que l'on peut apercevoir dans la série ; la façon dont le document arrive jusqu'à la Maison-Blanche et comment le Président oppose son veto à un texte ${ }^{11}$. Quand le Congrès décide d'infliger un blâme au Président pour avoir menti à propos de sa maladie, ce sont ici aussi les dessous de l'affaire qui sont mis en lumière : les discussions secrètes entre le conseil de la majorité et Leo McGarry ${ }^{12}$. De même et de manière, peut-être, plus explicite encore, les discours sur l'état de l'Union prononcés par le Président Bartlet ne sont presque jamais portés à l'écran alors que leur préparation et même les instants qui les précèdent, eux, le sont ${ }^{13}$. En cela, The West Wing offre des images différentes du phénomène constitutionnel. En effet, ce ne sont pas les aspects connus - médiatisés ou publics - de la présidence qui sont montrés mais plutôt ce que le citoyen, dans la réalité, ne peut observer : les «à-côtés » ou, plus exactement, ce 
qui se passe en amont et en aval de l'élément médiatisé (la prise de décision et les arguments invoqués, les conséquences de la décision et les responsabilités qui en découlent, ou encore les simples instants qui précèdent et succèdent un événement d'ordre constitutionnel).

Toutefois, les épisodes de la série ne se contentent pas de porter à l'écran des questions constitutionnelles, ils en expliquent également les rouages.

\section{Des explications}

17 Par sa qualité, sa richesse, le nombre de situations constitutionnelles abordées, la série est un formidable instrument d'appréhension du droit constitutionnel des États-Unis, de ce qu'est une Constitution, de ce pour quoi elle est faite et de la manière dont elle est appliquée et mise en œuvre. Bien sûr, la série ne fournit pas de réponse complète et exhaustive - ce n'est d'ailleurs ni son but, ni son objet - mais elle propose des éléments de réponse, des éléments d'information qui permettent néanmoins de saisir l'essentiel des fonctions de la Constitution américaine.

\section{La Constitution, garante des droits et libertés individuelles}

The West Wing met ainsi en évidence la fonction de garantie des droits de la Constitution. Dans la tradition américaine et dès le XVIII ${ }^{e}$ siècle, la Constitution doit être la loi suprême de l'État et doit servir à garantir les droits et libertés des individus contre l'arbitraire d'un pouvoir trop puissant voire tout-puissant. Cela s'inscrit dans le constitutionnalisme développé, notamment, par Jefferson, Paine ou Madison ${ }^{14}$. Cette fonction de garantie des droits, on la retrouve et on la comprend dans la série.

Dès la première saison, lorsqu'il s'agit de désigner un nouveau membre de la Cour suprême et lorsque le choix du Président Bartlet se porte sur Peyton Cabot Harrison (avant la nomination du juge Mendoza), les scénaristes mettent en évidence l'importance de la protection des droits et libertés des individus par le texte constitutionnel ${ }^{15}$. Le juge Harrison affirme ainsi très clairement que "les lois doivent émaner de la Constitution " (1.9). Cela a deux conséquences. La première est que la Constitution est bien présentée comme la loi suprême du pays (comme le dit l'article 6 de la Constitution elle-même). Quelques répliques plus tard, Sam Seaborn souligne l'importance de la protection constitutionnelle des droits et libertés :

Il ne s'agit pas seulement de l'avortement. Il s'agit des vingt prochaines années. Les années 20 et les années 30 ont été celles du rôle du gouvernement. Les années 50 et 60 furent celles des droits civiques. Les vingt prochaines années seront celles du droit à la vie privée. Je parle d'internet. Je parle des téléphones portables. Je parle des données médicales et de qui est homosexuel ou de qui ne l'est pas. Et, dans un pays né sur la volonté d'être libre, que pourrait-il y avoir de plus fondamental que cela ? ${ }^{16}$

Cette réflexion place alors la série, et c'est la seconde conséquence, au cœur des questions qui animent notamment les constitutionnalistes américains quant au rôle de la Cour suprême dans la protection des droits ${ }^{17}$. L'interrogation porte ainsi sur ce que la fonction constitutionnelle de garantie des droits implique aujourd'hui en termes de protection des droits fondamentaux, et c'est le débat entre les tenants de l'original intent et les partisans 
de la living interpretation qui s'instaure entre les personnages et qui est présenté au spectateur.

21 L'opposition entre les deux protagonistes est claire : le juge Harrison soutient que le droit à la vie privée ne figure pas, en tant que tel, dans la Constitution de sorte qu'un juge ne pourrait en assurer le respect au nom d'une disposition constitutionnelle tandis que Sam estime que le droit à la vie privée est un droit que la Constitution doit protéger et dont certains éléments sont, selon lui, présents et intégrés au sein de plusieurs amendements (il cite particulièrement les $\mathrm{III}^{\mathrm{e}}$, $\mathrm{IV}^{\mathrm{e}}$ et $\mathrm{V}^{\mathrm{e}}$ amendements).

22 En quelques phrases seulement, par la qualité et la puissance de ses dialogues, cet épisode résume l'une des fonctions de la Constitution. La Constitution est la loi suprême, la loi fondamentale de l'État, celle que tous les autres textes doivent respecter. C'est d'ailleurs dans ce texte-là, dans la Constitution et son interprétation, que sont définis les droits et libertés des individus. À travers cet épisode, c'est cette fonction de garantie des droits qui est soulignée et qui peut être perçue par les spectateurs.

\section{La Constitution, garante des institutions}

23 Autre fonction mise en évidence par la série: la fonction institutionnelle de la Constitution. Une constitution est faite pour organiser les pouvoirs et les institutions de l'État. The West Wing nous permet de comprendre, par exemple, la séparation des pouvoirs et la répartition des compétences entre le pouvoir exécutif (le Président) et le pouvoir législatif (le Congrès) en matière budgétaire. Les épisodes 7 et 8 de la saison 5 , consacrés à l'élaboration du budget fédéral, illustrent particulièrement cette répartition.

Une première discussion entre le Vice-Président et le Secrétaire général de la MaisonBlanche permet de comprendre qu'il appartient au Congrès d'élaborer le budget et que le Président peut simplement y opposer son veto. C'est le Vice-Président qui présente cette répartition des compétences en prenant appui non seulement sur les dispositions de la Constitution mais aussi sur la volonté des Pères fondateurs et, en particulier, James Madison. Une seconde discussion, à la fin de l'épisode 5.8, entre le Président Bartlet et le Président de la Chambre des Représentants Haffley revient sur cette répartition des compétences entre le Président et le Congrès en soulignant alors l'affrontement qui existe entre les deux pouvoirs. Le Président de la Chambre affirme que «la Constitution confie au Congrès le soin d'élaborer et d'adopter le budget »; le Président Bartlet le coupe immédiatement pour lui rappeler que la Constitution donne aussi au Président un droit de veto ${ }^{18}$.

La série explique très simplement à quoi sert, principalement, une Constitution: la protection des droits et libertés, l'organisation et la répartition des pouvoirs.

\section{Une série pédagogique}

Par ailleurs, The West Wing parvient à rendre compte de manière assez exacte de mécanismes particulièrement techniques que les spectateurs ne maîtrisent pas nécessairement mais que le scénario doit néanmoins leur permettre de saisir. Martin Winckler observe ainsi un procédé explicatif récurrent de la série: «chacun [des personnages] a, à son tour, l'occasion de se faire la voix du spectateur (du citoyen) pour dire qu'il ne comprend pas ce qui se passe, et obtenir ainsi qu'on nous/qu'on le lui explique $^{19}$. » De cette manière, les conversations entre les personnages de la série servent 
aussi bien le déroulement de l'intrigue que l'explication des mécanismes institutionnels qui sont parfois subtils mais dont la compréhension est nécessaire aux spectateurs.

Le sixième épisode de la saison 2 met, par exemple, en évidence un aspect du droit parlementaire américain: ce que l'on appelle les lame-duck sessions. Les membres du Congrès des États-Unis sont élus au mois de novembre mais les nouveaux Sénateurs et Représentants ne prennent leurs fonctions qu'au mois de janvier. Par conséquent, durant cette période qui sépare les élections de l'installation des nouveaux élus (entre novembre et janvier), des élus sortants siègent au sein des Chambres. Cette session parlementaire entre novembre et janvier - est alors dénommée la lame-duck session, la session des « canards boiteux ».

L'épisode consiste principalement en une interrogation sur le caractère représentatif du Congrès des sortants et sur la possibilité - constitutionnelle mais aussi éthique - pour le Président de demander un vote du Congrès sortant sur une question à propos de laquelle les nouveaux élus ont déjà fait savoir, au cours de la campagne électorale notamment, qu'ils y étaient plutôt défavorables ${ }^{20}$. Ce qui est alors intéressant à observer, c'est que l'épisode permet aux spectateurs de comprendre les grands enjeux de la question: en quoi consiste la lame-duck session, les arguments en faveur ou en défaveur d'une sollicitation des élus sortants. L'effort pédagogique est certain.

Pour autant, l'explication du mécanisme n'est pas complète. Certains éléments comme les raisons historiques des sessions post-électorales ou des éléments plus techniques encore ne sont pas développés. La série permet donc de comprendre le mécanisme mais, en réalité, seulement ce qu'il faut pour que l'intrigue nouée autour de la question puisse être comprise et appréciée par ceux qui regardent l'épisode. Le mécanisme constitutionnel est, avant tout, un prétexte dramaturgique. La richesse pédagogique de la série se trouve pourtant là. Le scénario n'est jamais appauvri pour qu'il soit compréhensible; bien au contraire, la narration est enrichie pour permettre à chacun de se voir expliquer le fonctionnement des institutions.

30 Avec beaucoup d'intelligence, The West Wing explique aussi bien aux spectateurs ce qu'est une Constitution et ce à quoi elle sert que des mécanismes constitutionnels techniques, comment on les met en œuvre et quels en sont les enjeux. The West Wing nous permet de comprendre tout cela car elle nous en offre une explication in vivo. C'est pour cela qu'elle peut être un extraordinaire instrument d'appréciation, de compréhension et, finalement, d'initiation au droit constitutionnel.

31 Mais, la série est avant tout une œuvre de divertissement. Elle nous encourage alors à imaginer le phénomène constitutionnel en en forgeant ou en en proposant des représentations.

\section{Des représentations}

32 Par les images qu'elle propose de la Constitution et du droit constitutionnel et les explications qu'elle en donne, la série offre un reflet de la réalité. Edgar Morin le disait à propos du cinéma : «le cinéma majore le réel. [...] C'est une merveille anthropologique. Très précisément en cette adéquation à projeter en spectacle une image perçue comme reflet exact de la réalitée ${ }^{11}$.» The West Wing participe alors à la fabrication d'un certain nombre de représentations sociales, d'images plus ou moins exactes et plus ou moins précises de ce qu'est la Constitution et le droit constitutionnel des États-Unis. 


\section{Un récit inspiré par la réalité} en ce que la fiction prolonge parfois la réalité. Le douzième épisode de la première saison est, à cet égard, significatif ${ }^{25}$. Le Président Bartlet et son équipe s'interrogent et discutent une phrase prévue dans le discours sur l'état de l'Union que le Président doit délivrer quelques heures plus tard. Or, cette phrase - « the era of big governement is over » - est une phrase réellement prononcée par le Président Clinton dans son propre discours sur l'état de l'Union en 1996. Les scénaristes, à travers l'épisode de la série, critiquent le discours réel et en proposent une réécriture qui transforme le récit présidentiel. Alors que le Président Clinton avait effectivement prononcé cette phrase, le Président Bartlet, lui, la retire. On peut ainsi souligner avec Marjolaine Boutet que « les premiers épisodes de The West Wing se présentent très clairement aux Américains comme ce qu'aurait pu être la politique des Démocrates au pouvoir à la fin des années 1990, sans le parfum de scandale et la force d'opposition d'un Congrès républicain ${ }^{26}$. »

\section{Une représentation idéalisée}

The West Wing prolonge, en outre, une réalité qu'elle idéalise. Cela vaut pour la politique menée par l'administration Bartlet mais aussi pour les mécanismes constitutionnels qui sont mis en œuvre. L'épisode consacré au filibuster ${ }^{27}$ - ce mécanisme consistant pour un sénateur à prendre la parole le plus longtemps possible afin de retarder l'organisation 
d'un vote sur une mesure avec laquelle il est en désaccord - en propose, par exemple, une représentation largement positive comme c'était déjà le cas dans le film de Franck Capra, Mr Smith Goes to Washington (1939), dans lequel James Stewart incarne un jeune sénateur idéaliste accusé à tort de corruption et qui, pour prouver son innocence, s'engage dans un filibuster qu'il mène jusqu'au bout de ses forces.

Dans l'épisode de la série, le spectateur ne comprend pas immédiatement pourquoi le sénateur Stackhouse se lance dans une telle procédure. En revanche, parce que plusieurs conseillers du Président - Sam, C.J. et Josh - en profitent pour écrire à leurs proches, le mécanisme constitutionnel ou, plus exactement, le mécanisme parlementaire du filibuster est, lui, expliqué. On comprend grâce à Josh Lyman l'origine du mot ; on comprend grâce à C.J. Gregg les conséquences de l'obstruction parlementaire en cours ainsi que son caractère un peu vain; on comprend aussi grâce à Sam Seaborn pourquoi ce filibuster paralyse, pour un temps au moins, l'administration Bartlet. Ce sont donc bien les conversations, les dialogues qui permettent d'expliquer aux spectateurs des éléments de procédure. Mais surtout, dans le film de Capra comme dans The West Wing, le filibuster apparait comme un instrument vertueux, un ultime moyen pour défendre ses convictions, son honneur et ses idées. Dans la série, le sénateur Stackhouse se bat pour le développement des aides financières en faveur de la recherche médicale contre l'autisme.

Or, la réalité constitutionnelle est un peu différente et l'obstruction parlementaire aux États-Unis n'est pas toujours aussi vertueuse. Dans la période récente, la multiplication des filibusters a très grandement ralenti le fonctionnement même du Sénat américain, notamment en ce qui concerne les nominations exigeant une confirmation de la part de ses membres, ce qui a d'ailleurs conduit à une réforme de la procédure d'obstruction ${ }^{28}$. La question est abordée dans The West Wing mais pas dans l'épisode principalement consacré au filibuster ${ }^{29}$. À cela, il faut encore ajouter que les débats réels, politiques et constitutionnels, autour du filibuster invoquent très souvent la représentation cinématographique de ce mécanisme pour souligner son importance démocratique, sa nécessité en tant que droit de l'opposition et en tant que droit pour chacun d'exprimer son opinion. Le débat constitutionnel réel est alors nourri par l'image idéalisée que le cinéma et les séries télévisées donnent, en l'occurrence, du Sénat et de ses procédures.

\section{Conclusion}

40 La participation à la construction de ces images plus ou moins exactes et plus ou moins précises quant à ce qu'est le droit constitutionnel - autrement dit la participation à la construction de représentations sociales du droit constitutionnel - résulte donc d'une exagération de la réalité. Sabine Chalvon-Demersay l'avait déjà souligné à propos de la série Urgences. La fiction propose une exagération des situations rencontrées par rapport à la réalité tout en s'appuyant sur « la précision technique de tout ce qui [renvoyait] à la partie proprement médicale ${ }^{30}$.» Le procédé est identique dans The West Wing en ce qui concerne le droit constitutionnel.

41 La force de la série est alors de rendre cependant plausible l'extraordinaire et de faire ainsi entrer dans la vie quotidienne des spectateurs des représentations de ce qu'est la Constitution, la vie institutionnelle et le droit constitutionnel. À travers The West Wing apparait même une forme de mythe constitutionnel. Dans un ouvrage consacré à la naissance de la Constitution française de 1958, Bastien François écrit: «on ne 
comprendrait rien à ce que les acteurs investissent dans le travail constituant si l'on perdait de vue qu'une Constitution est d'abord une sorte de totem, marqueur ou emblème de la nécessité qui conduit une communauté à se donner à voir comme telle en proclamant ce qui l'unit et ce qui la sépare des autres ${ }^{31}$.» Or, c'est aussi ce rapport particulier à la Constitution que la série parvient à souligner. Même si, bien sûr, tout cela ne doit pas être exagéré, il n'en reste pas moins que The West Wing est un formidable outil d'initiation au droit constitutionnel et un très puissant vecteur de sa diffusion.

\section{BIBLIOGRAPHIE}

BOUTET Marjolaine, «Le Président des États-Unis, héros de séries télévisées. La figure présidentielle dans les séries américaines récentes », Le Temps des Médias, n 10, 2008, p. 156 CHALVON-DEMERSAY Sabine, « La confusion des sentiments. Une enquête sur la série télévisée Urgences », Réseaux, n 95, 1999, p. 237

CONNIL Damien, « Le discours sur l'état de l'Union, The West Wing et l'imaginaire constitutionnel ", Pouvoirs, n 148, 2014, p. 151

CONNIL Damien, «Quand le vote du budget se transforme en thriller... À propos de deux épisodes de The West Wing ", in TOUZEIL-DIVINA Mathieu (dir.), Le Parlement aux écrans !, Le Mans, Éd. L'Épitoge - Lextenso, 2013, p. 141

ESQUENAZI Jean-Pierre, Les Séries télévisées, l'avenir du cinéma ?, Paris, Armand Colin, 2010

FRANÇOIS Bastien, Naissance d'une Constitution : la Ve République, 1958-1962, Paris, Presses de Sciences Po, 1996

GIRARD Charles, «The world can move or not, by changing some words : La parole politique en fiction dans The West Wing ", Revue de recherche en civilisation américaine [En ligne], 2/2010, http:// rrca.revues.org/index310.html

MORIN Edgar, Le cinéma ou l'homme imaginaire, Essai d'anthropologie, Paris, Ed. de Minuit, 1958, réédition Éd. Gonthier, 1965

WINCKLER Martin, «Les coulisses du pouvoir », Le Monde Diplomatique, 23 août 2003, disponible sur MartinWinckler.com

WINCKLER Martin, Petit éloge des séries télé, Paris, Gallimard, coll. Folio, 2012

\section{NOTES}

1. Voir notamment "The Short List» (1.9); "On the Day Before » (3.4); "The Stackhouse Filibuster » (2.17) ; «Inauguration » (4.14 et 4.15) ; «Separation of Powers » (5.7) et "Shutdown » (5.8); « The Supremes » (5.17); « The Benign Prerogative » (5.11).

2. «The Short List » (1.9)

3. « 100,000 Airplanes » (3.11) 
4. Pour de plus amples développements à partir du même exemple, voir Damien Connil, «Quand le vote du budget se transforme en thriller... À propos de deux épisodes de The West Wing ", in Mathieu Touzeil-Divina (dir.), Le Parlement aux écrans !, Éd. L'Épitoge - Lextenso, 2013, p. 141.

5. L'expression est empruntée à Marjolaine Boutet in "Le Président des États-Unis, héros de séries télévisées. La figure présidentielle dans les séries américaines récentes ", Le Temps des Médias, 2008, n 10, p. 156.

6. Ibid.

7. Voir notamment « He shall from time to time... » (1.12); «Bartlet's Third State of the Union » et «The War at Home» (2.13 et 2.14) ; «100,000 Airplanes » (3.11) ; "The Benign Prerogative » (5.11) et «Slow News Day » (5.12) ; « 365 days » (6.12) ; « The Red Mass » (4.4); «Inauguration » (4. 14 et 4.15).

8. Voir entre autres: «Post Hoc, Ergo Propter Hoc » (1.2) et «A Proportional Response» (1.3); « Gone Quiet» (3.6), « We Killed Yamamoto » (3.20) et « Posse Comitatus » (3.21) ; « Inauguration: Over There » (4.15), « The California $47^{\text {th }}$ » (4.16) et « Red Haven's on Fire » (4.17).

9. Charles Girard, "The world can move or not, by changing some words: La parole politique en fiction dans The West Wing", Revue de recherche en civilisation américaine [En ligne], 2/2010, disponible sur http://rrca.revues.org/index310.html, § 24.

10. «Twenty Five » (5.23).

11. « On the Day Before» (3.4)

12. « H. Con-172» (3.10).

13. Voir particulièrement « 100,000 Airplanes » (3.11).

14. Sur la notion de constitutionnalisme, voir Philippe Raynaud, "Constitutionnalisme » in D. Alland et S. Rials, Dictionnaire de la culture juridique, PUF, 2003.

15. « The Short List » (1.9)

16. Souligné par l'auteur.

17. Pour une présentation des enjeux de la question, on peut utilement se référer à R. Dworkin, «Controverse constitutionnelle », Pouvoirs, 1991, n 59, p. 5.

18. Voir Damien Connil, art. préc., in $\mathrm{M}$. Touzeil-Divina (dir.), Le Parlement aux écrans!, Éd. L’Épitoge - Lextenso, 2013, p. 142-143.

19. Martin Winckler, «Les coulisses du pouvoir », Le Monde Diplomatique, 23 août 2003. Disponible sur MartinWinckler.com.

20. «The Lame Duck Congress » (2.6)

21. Edgar Morin, Le cinéma ou l'homme imaginaire, Essai d'anthropologie, Paris, Éd. de Minuit, 1958, réédition Éd. Gonthier, 1965, p. 40.

22. Girard, op.cit., § 18.

23. Jean-Pierre Esquenazi, Les Séries télévisées, l'avenir du cinéma?, Paris, Armand Colin, 2010, p. 182.

24. Martin Winckler, Petit éloge des séries télé, Paris, Gallimard, coll. Folio, 2012, p. 20.

25. «He Shall from Time to Time » (1.12). Pour une analyse plus détaillée, v. Damien Connil, « Le discours sur l'état de l'Union, The West Wing et l'imaginaire constitutionnel », Pouvoirs, 2014, n - 148 , p. 151.

26. Boutet, op.cit., p. 158.

27. «The Stackhouse Filibuster» (2.17)

28. Sur le filibuster, voir Murielle Mauguin-Helgeson, "Représentations et mise en scène de l'obstruction parlementaire ", in Mathieu Touzeil-Divina (dir.), Le Parlement aux écrans !, L'Épitoge, 2013, p. 151 et s. et Damien Connil, «La petite révolution américaine, Quand le Sénat tente de limiter l'obstruction parlementaire », Constitutions, 2014, p. 38.

29. « Eppur Si Muove » (5.16)

30. Sabine Chalvon-Demersay, «La confusion des sentiments. Une enquête sur la série télévisée Urgences ", Réseaux, 1999, n 95, p. 237 et spécialement pp. 255-257. 
31. Bastien François, Naissance d'une Constitution : la V République, 1958-1962, Presses de Sciences Po, 1996, p. 59, souligné par l'auteur.

\section{RÉSUMÉS}

L'étude propose d'analyser les images, les explications et les représentations que The West Wing offre de la Constitution et du droit constitutionnel afin d'apprécier ce que la série nous permet de comprendre et d'imaginer de la réalité du phénomène constitutionnel aux États-Unis.

This article analyzes the representation of the Constitution and constitutional law in The West Wing, in order to show that the TV series makes it possible to understand and imagine constitutional issues in the United States.

\section{INDEX}

Mots-clés : À la Maison Blanche, West Wing (The), politique, série télévisée, constitution, droit constitutionnel, États-Unis, imaginaire, représentation

Keywords : West Wing (The), politics, TV series, constitution, constitutional law, United States, representations, images

\section{AUTEUR}

\section{DAMIEN CONNIL}

Damien Connil est chargé de recherches au CNRS (UMR 7318, Université de Pau et des Pays de l'Adour, Droit public comparé, Droit international et Droit européen).

Damien Connil is a CNRS researcher (UMR 7318, Pau and Pays de l'Adour University, Compared Public Law, International and European Law). 